\title{
Isogenies and the Discrete Logarithm Problem in Jacobians of Genus 3 Hyperelliptic Curves
}

\author{
Benjamin Smith \\ INRIA Saclay-île-de-France \\ Laboratoire d'Informatique de l'École polytechnique (LIX) \\ 91128 Palaiseau Cedex, France \\ smith@lix.polytechnique.fr
}

\begin{abstract}
We describe the use of explicit isogenies to translate instances of the Discrete Logarithm Problem from Jacobians of hyperelliptic genus 3 curves to Jacobians of non-hyperelliptic genus 3 curves, where they are vulnerable to faster index calculus attacks. We provide explicit formulae for isogenies with kernel isomorphic to $(\mathbb{Z} / 2 \mathbb{Z})^{3}$ (over an algebraic closure of the base field) for any hyperelliptic genus 3 curve over a field of characteristic not 2 or 3 . These isogenies are rational for a positive fraction of all hyperelliptic genus 3 curves defined over a finite field of characteristic $p>3$. Subject to reasonable assumptions, our constructions give an explicit and efficient reduction of instances of the DLP from hyperelliptic to non-hyperelliptic Jacobians for around $18.57 \%$ of all hyperelliptic genus 3 curves over a given finite field.
\end{abstract}

\section{Introduction}

After the great success of elliptic curves in cryptography, researchers have naturally been drawn to their higher-dimensional generalizations: Jacobians of highergenus curves. Curves of genus 1 (elliptic curves), 2, and 3 are widely believed to offer the best balance of security and efficiency. This article is concerned with the security of curves of genus 3 .

There are two classes of curves of genus 3: hyperelliptic and non-hyperelliptic. Each class has a distinct geometry: the canonical morphism of a hyperelliptic curve is a double cover of a curve of genus 0 , while the canonical morphism of a non-hyperelliptic curve of genus 3 is an isomorphism to a nonsingular plane quartic curve. A hyperelliptic curve cannot be isomorphic (or birational) to a nonhyperelliptic curve. From a cryptological point of view, the Discrete Logarithm Problem (DLP) in Jacobians of hyperelliptic curves of genus 3 over $\mathbb{F}_{q}$ may be solved in $\widetilde{O}\left(q^{4 / 3}\right)$ group operations, using the index calculus algorithm of Gaudry, Thomé, Thériault, and Diem [6]. Jacobians of non-hyperelliptic curves of genus 3 over $\mathbb{F}_{q}$ are amenable to Diem's index calculus algorithm [3], which requires only $\widetilde{O}(q)$ group operations to solve the DLP (for comparison, Pollard/babystep-giant-step methods require $\widetilde{O}\left(q^{3 / 2}\right)$ group operations to solve the DLP in Jacobians of genus 3 curves over $\mathbb{F}_{q}$ ). The security of non-hyperelliptic genus 3 curves is therefore widely held to be lower than that of their hyperelliptic cousins. 
Our aim is to provide a means of efficiently translating DLPs from Jacobians of hyperelliptic genus 3 curves to Jacobians of non-hyperelliptic curves, where faster index calculus is available. We do this by constructing explicit isogenies of Jacobians: surjective homomorphisms, with finite kernel, from hyperelliptic to non-hyperelliptic Jacobians. The kernels of our isogenies will intersect trivially with any subgroup of cryptographic interest, and so the isogenies will restrict to isomorphisms of DLP subgroups.

Specifically, let $H$ be a hyperelliptic curve of genus 3 over a finite field of characteristic $p>3$. Suppose the Jacobian $J_{H}$ of $H$ contains a subgroup $S$ isomorphic to $(\mathbb{Z} / 2 \mathbb{Z})^{3}$ (over an algebraic closure of the base field), generated by differences of Weierstrass points. If the 2-Weil pairing restricts trivially to $S$, then there exists an isogeny with kernel $S$ from $J_{H}$ to a principally polarized abelian variety $A$. Using Recillas' trigonal construction [12, $A$ may be realized as the Jacobian of a genus 3 curve $X$. This construction appears to be due to Donagi and Livné [5] our contribution, aside from the cryptological application, is to provide explicit formulae for the curve $X$ and the isogeny. Naïve moduli space dimension arguments suggest that there is an overwhelming probability that $X$ will be non-hyperelliptic, and thus explicitly isomorphic to a nonsingular plane quartic curve $C$. We therefore obtain an explicit isogeny $\phi: J_{H} \rightarrow J_{C}$ with kernel $S$. If $\phi$ is defined over $\mathbb{F}_{q}$, then it maps $J_{H}\left(\mathbb{F}_{q}\right)$ into $J_{C}\left(\mathbb{F}_{q}\right)$, where Diem's $\widetilde{O}(q)$ index calculus is available. Given points $P$ and $Q=[n] P$ of odd order in $J_{H}\left(\mathbb{F}_{q}\right)$, we can solve the DLP (that is, recovering $n$ from $P$ and $Q$ ) in $J_{C}\left(\mathbb{F}_{q}\right)$, using

$$
Q=[n] P \Longrightarrow \phi(Q)=[n] \phi(P) .
$$

There are several caveats to our approach, besides the requirement of a subgroup $S$ as described above. First, it does not apply in characteristic 2 or 3 . In characteristic 2, the subgroup $S$ is the kernel of a verschiebung, so $X$ is necessarily hyperelliptic. In characteristic 3 , we cannot use the trigonal construction. Second, in order to obtain an advantage with index calculus on $X$ over $H$, the isogeny must be defined over $\mathbb{F}_{q}$ and $X$ must be non-hyperelliptic. We show in 88 that, subject to some reasonable assumptions, given a hyperelliptic curve $H$ of genus 3 over a sufficiently large finite field, our algorithms succeed in giving an explicit rational isogeny from $J_{H}$ to a non-hyperelliptic Jacobian with probability $\approx 0.1857$. In particular, instances of the DLP can be solved in $\widetilde{O}(q)$ group operations for around $18.57 \%$ of all Jacobians of hyperelliptic curves of genus 3 over a finite field of characteristic $p>3$.

Our results have a number of interesting implications for curve-based cryptography, at least for curves of genus 3. First, the difficulty of the DLP in a subgroup $G$ of $J_{H}$ depends not only on the size of the subgroup $G$, but upon the existence of other rational subgroups of $J_{H}$ that can be used to form quotients. Second, the security of a given hyperelliptic genus 3 curve depends significantly upon the factorization of its hyperelliptic polynomial. Neither of these results has any parallel in genus 1 or 2 .

After reviewing some standard definitions for hyperelliptic curves in 92 we define the kernels of our isogenies in $\$ 3$. In $\$ 4$ \$5 and $\$ 6$, we describe and derive 
explicit formulae for the trigonal construction, which is our main tool for constructing isogenies. After giving an example in \$7] we compute (heuristically) the expectation that the methods of this article will compute a rational isogeny for a randomly chosen curve in $\$ 8$. Finally, in 99 we briefly describe some of the problems involved in generalizing these methods.

\section{A Note on the Base Field}

We will work over $\mathbb{F}_{q}$ throughout this article, where $q$ is a power of a prime $p>3$. We let $\mathcal{G}$ denote the Galois group $\operatorname{Gal}\left(\overline{\mathbb{F}}_{q} / \mathbb{F}_{q}\right)$, which is (topologically) generated by the $q^{\text {th }}$ power Frobenius map. Some of the theory of this article carries over to fields of characteristic zero: in particular, the results of $\$ 5$ and $\sqrt[6]{6}$ are valid over fields of characteristic not 2 or 3 .

\section{Notation and Conventions for Hyperelliptic Curves}

We assume that we are given a hyperelliptic curve $H$ of genus 3 over $\mathbb{F}_{q}$, and that the Jacobian $J_{H}$ of $H$ is absolutely simple. We will use both an affine model

$$
H: y^{2}=F(x)
$$

where $F$ is a squarefree polynomial of degree 7 or 8 , and a weighted projective plane model

$$
H: w^{2}=\widetilde{F}(u, v)
$$

for $H$ (where $u, v$, and $w$ have weights 1,1 , and 4 , respectively). The coordinates of these models are related by $x=u / v$ and $y=w / v^{4}$. The polynomial $\widetilde{F}$ is squarefree of total degree 8, with $\widetilde{F}(u, v)=v^{8} F(u / v)$ and $\widetilde{F}(x, 1)=F(x)$. We emphasize that $F$ need not be monic. By a randomly chosen hyperelliptic curve, we mean the hyperelliptic curve defined by $w^{2}=\widetilde{F}(u, v)$, where $\widetilde{F}$ is a uniformly randomly chosen squarefree homogenous bivariate polynomial of degree 8 over $\mathbb{F}_{q}$. The canonical hyperelliptic involution $\iota$ of $H$ is defined by $(x, y) \mapsto(x,-y)$ in the affine model, $(u: v: w) \mapsto(u: v:-w)$ in the projective model, and induces the negation map $[-1]$ on $J_{H}$. The quotient $\pi: H \rightarrow \mathbb{P}^{1} \cong$ $H /\langle\iota\rangle$ sends $(u: v: w)$ to $(u: v)$ in the projective model, and $(x, y)$ to $x$ in the affine model (where it maps onto the affine patch of $\mathbb{P}^{1}$ where $v \neq 0$ ).

To compute in $J_{H}$, we fix an isomorphism from $J_{H}$ to the group of degreezero divisor classes on $H$, denoted $\operatorname{Pic}^{0}(H)$. Recall that divisors are formal sums of points in $H\left(\overline{\mathbb{F}_{q}}\right)$, and if $D=\sum_{P \in H} n_{P}(P)$ is a divisor, then $\sum_{P \in H} n_{P}$ is the degree of $D$. We say $D$ is principal if $D=\operatorname{div}(f):=\sum_{P \in H} \operatorname{ord}_{P}(f)(P)$ for some function $f$ on $H$, where $\operatorname{ord}_{P}(f)$ denotes the number of zeroes (or the negative of the number of poles) of $f$ at $P$. Since $H$ is complete, every principal divisor has degree 0 . The group $\operatorname{Pic}^{0}(H)$ is defined to be the group of divisors of degree 0 modulo principal divisors; the equivalence class of a divisor $D$ is denoted by $[D]$. 


\section{The Kernel of the Isogeny}

The eight points of $H\left(\overline{\mathbb{F}_{q}}\right)$ where $w=0$ are called the Weierstrass points of $H$. Each Weierstrass point $W$ corresponds to a linear factor $L_{W}=v(W) u-u(W) v$ of $\widetilde{F}$. If $W_{1}$ and $W_{2}$ are Weierstrass points, then $2\left(W_{1}\right)-2\left(W_{2}\right)=\operatorname{div}\left(L_{W_{1}} / L_{W_{2}}\right)$, so $2\left[\left(W_{1}\right)-\left(W_{2}\right)\right]=0$; hence $\left[\left(W_{1}\right)-\left(W_{2}\right)\right]$ corresponds to an element of $J_{H}[2]\left(\overline{\mathbb{F}_{q}}\right)$ (the two-torsion subgroup of $J_{H}$ : that is, the kernel of multiplication by two $)$. In particular, $\left[\left(W_{1}\right)-\left(W_{2}\right)\right]=\left[\left(W_{2}\right)-\left(W_{1}\right)\right]$, so the divisor class $\left[\left(W_{1}\right)-\left(W_{2}\right)\right]$ corresponds to the pair $\left\{W_{1}, W_{2}\right\}$ of Weierstrass points, and hence to the quadratic factor $L_{W_{1}} L_{W_{2}}$ of $\widetilde{F}$.

Proposition 1. To every $\mathcal{G}$-stable partition of the eight Weierstrass points of $H$ into four disjoint pairs, we may associate an $\mathbb{F}_{q}$-rational subgroup of $J_{H}[2]\left(\overline{\mathbb{F}_{q}}\right)$ isomorphic to $(\mathbb{Z} / 2 \mathbb{Z})^{3}$.

Proof. Let $\left\{\left\{W_{1}^{\prime}, W_{1}^{\prime \prime}\right\},\left\{W_{2}^{\prime}, W_{2}^{\prime \prime}\right\},\left\{W_{3}^{\prime}, W_{3}^{\prime \prime}\right\},\left\{W_{4}^{\prime}, W_{4}^{\prime \prime}\right\}\right\}$ be a partition of the Weierstrass points of $H$ into four disjoint pairs. Each pair $\left\{W_{i}^{\prime}, W_{i}^{\prime \prime}\right\}$ corresponds to the two-torsion divisor class $\left[\left(W_{i}^{\prime}\right)-\left(W_{i}^{\prime \prime}\right)\right]$ in $J_{H}[2]\left(\overline{\mathbb{F}_{q}}\right)$. We associate the subgroup $S:=\left\langle\left[\left(W_{i}^{\prime}\right)-\left(W_{i}^{\prime \prime}\right)\right]: 1 \leq i \leq 4\right\rangle$ to the partition. Observe that

$$
\sum_{i=1}^{4}\left[\left(W_{i}^{\prime}\right)-\left(W_{i}^{\prime \prime}\right)\right]=\left[\operatorname{div}\left(w / \prod_{i=1}^{4} L_{W_{i}^{\prime \prime}}\right)\right]=0
$$

this is the only relation on the classes $\left[\left(W_{i}^{\prime}\right)-\left(W_{i}^{\prime \prime}\right)\right]$, so $S \cong(\mathbb{Z} / 2 \mathbb{Z})^{3}$. The action of $\mathcal{G}$ on $J_{H}[2]\left(\overline{\mathbb{F}_{q}}\right)$ corresponds to its action on the Weierstrass points, so if the partition is $\mathcal{G}$-stable, then the subgroup $S$ is $\mathcal{G}$-stable.

Remark 1. Requiring the pairs of points to be disjoint ensures that the associated subgroup is 2-Weil isotropic. This is necessary for the quotient by the subgroup to be an isogeny of principally polarized abelian varieties (see \$9).

Remark 2. By "an $\mathbb{F}_{q}$-rational subgroup of $J_{H}[2]\left(\overline{\mathbb{F}_{q}}\right)$ isomorphic to $(\mathbb{Z} / 2 \mathbb{Z})^{3}$ ", we mean a $\mathcal{G}$-stable subgroup that is isomorphic to $(\mathbb{Z} / 2 \mathbb{Z})^{3}$ over $\overline{\mathbb{F}_{q}}$. We emphasize that the elements of the subgroup need not be $\mathbb{F}_{q}$-rational themselves.

Definition 1. We call the subgroups corresponding to partitions of the Weierstrass points of $H$ as in Proposition 1 tractable subgroups. We let $\mathcal{S}(H)$ denote the set of all $\mathbb{F}_{q}$-rational tractable subgroups of $J_{H}[2]\left(\overline{\mathbb{F}_{q}}\right)$.

Remark 3. Not every subgroup of $J_{H}[2]\left(\overline{\mathbb{F}_{q}}\right)$ that is the kernel of an isogeny of Jacobians is a tractable subgroup. For example, if $W_{1}, \ldots, W_{8}$ are the Weierstrass points of $H$, then the subgroup

$$
\left\langle\left[\left(W_{1}\right)-\left(W_{i}\right)+\left(W_{j}\right)-\left(W_{k}\right)\right]:(i, j, k) \in\{(2,3,4),(2,5,6),(3,5,7)\}\right\rangle
$$

is maximally 2-Weil isotropic, and hence is the kernel of an isogeny of Jacobians (see \$9). However, this subgroup contains no nontrivial differences of Weierstrass points, and so cannot be a tractable subgroup. 
Computing $\mathcal{S}(H)$ is straightforward if we identify each tractable subgroup with its corresponding partition of Weierstrass points. Each pair $\left\{W_{i}^{\prime}, W_{i}^{\prime \prime}\right\}$ of Weierstrass points corresponds to a quadratic factor of $\widetilde{F}$. Since the pairs are disjoint, the corresponding quadratic factors are pairwise coprime, and hence form (up scalar multiples) a factorization of the hyperelliptic polynomial $\widetilde{F}$. We therefore have a correspondence of tractable subgroups, partitions of Weierstrass points into pairs, and sets of quadratic polynomials (up to scalar multiples):

$$
S \longleftrightarrow\left\{\left\{W_{i}^{\prime}, W_{i}^{\prime \prime}\right\}: 1 \leq i \leq 4\right\} \longleftrightarrow\left\{F_{1}, F_{2}, F_{3}, F_{4}\right\} \text {, where } \widetilde{F}=F_{1} F_{2} F_{3} F_{4} .
$$

Since the action of $\mathcal{G}$ on $J_{H}[2]\left(\overline{\mathbb{F}_{q}}\right)$ corresponds to its action on the set of Weierstrass points, the action of $\mathcal{G}$ on a tractable subgroup $S$ corresponds to the action of $\mathcal{G}$ on the corresponding set $\left\{F_{1}, F_{2}, F_{3}, F_{4}\right\}$. In particular, $S$ is $\mathbb{F}_{q}$-rational precisely when $\left\{F_{1}, F_{2}, F_{3}, F_{4}\right\}$ is fixed by $\mathcal{G}$. The factors $F_{i}$ are themselves defined over $\mathbb{F}_{q}$ precisely when the corresponding points of $S$ are $\mathbb{F}_{q}$-rational.

We can use this information to compute $\mathcal{S}(H)$. The set of pairs of Weierstrass points contains a $\mathcal{G}$-orbit $\left(\left\{W_{i_{1}}^{\prime}, W_{i_{1}}^{\prime \prime}\right\}, \ldots,\left\{W_{i_{n}}^{\prime}, W_{i_{n}}^{\prime \prime}\right\}\right)$ if and only if (possibly after exchanging some of the $W_{i_{k}}^{\prime}$ with the $\left.W_{i_{k}}^{\prime \prime}\right)$ either both $\left(W_{i_{1}}^{\prime}, \ldots, W_{i_{n}}^{\prime}\right)$ and $\left(W_{i_{1}}^{\prime \prime}, \ldots, W_{i_{n}}^{\prime \prime}\right)$ are $\mathcal{G}$-orbits or $\left(W_{i_{1}}^{\prime}, \ldots, W_{i_{n}}^{\prime}, W_{i_{1}}^{\prime \prime}, \ldots, W_{i_{n}}^{\prime \prime}\right)$ is a $\mathcal{G}$-orbit. Every $\mathcal{G}$-orbit of Weierstrass points corresponds to an $\mathbb{F}_{q}$-irreducible factor of $F$. Elementary calculations therefore yield the following useful lemma, as well as algorithms to compute all of the $\mathbb{F}_{q}$-rational tractable subgroups of $J_{H}[2]\left(\overline{\mathbb{F}_{q}}\right)$.

Lemma 1. Let $H: w^{2}=\widetilde{F}(u, v)$ be a hyperelliptic curve of genus 3 over $\mathbb{F}_{q}$. The cardinality of the set $\mathcal{S}(H)$ depends only on the degrees of the $\mathbb{F}_{q}$-irreducible factors of $\widetilde{F}$, and is described by the following table:

\begin{tabular}{|c|c|}
\hline Degrees of $\mathbb{F}_{q}$-irreducible factors of $\widetilde{F}$ & $\# \mathcal{S}(H)$ \\
\hline \hline$(8),(6,2),(6,1,1),(4,2,1,1)$ & 1 \\
\hline$(4,4)$ & 5 \\
\hline$(4,2,2),(4,1,1,1,1),(3,3,2),(3,3,1,1)$ & 3 \\
\hline$(2,2,2,1,1)$ & 7 \\
\hline$(2,2,1,1,1,1)$ & 9 \\
\hline$(2,1,1,1,1,1,1)$ & 15 \\
\hline$(2,2,2,2)$ & 25 \\
\hline$(1,1,1,1,1,1,1,1)$ & 105 \\
\hline Other & 0 \\
\hline
\end{tabular}

\section{The Trigonal Construction}

We will now briefly outline the theoretical aspects of constructing isogenies with tractable kernels. We will make the construction completely explicit in $\$ 5$ and $\sqrt{6}$.

Definition 2. Suppose $S=\left\langle\left[\left(W_{i}^{\prime}\right)-\left(W_{i}^{\prime \prime}\right)\right]: 1 \leq i \leq 4\right\rangle$ is a tractable subgroup. We say that a morphism $g: \mathbb{P}^{1} \rightarrow \mathbb{P}^{1}$ is a trigonal map for $S$ if $g$ has degree 3 and $g\left(\pi\left(W_{i}^{\prime}\right)\right)=g\left(\pi\left(W_{i}^{\prime \prime}\right)\right)$ for $1 \leq i \leq 4$. 
Given a trigonal map $g$, Recillas' trigonal construction [12] specifies a curve $X$ of genus 3 and a map $f: X \rightarrow \mathbb{P}^{1}$ of degree 4 . The isomorphism class of $X$ is independent of the choice of $g$. Theorem 1, due to Donagi and Livné, states that if $g$ is a trigonal map for $S$, then $S$ is the kernel of an isogeny from $J_{H}$ to $J_{X}$.

Theorem 1 (Donagi and Livné [5, §5]). Let $S$ be a tractable subgroup of $J_{H}[2]\left(\overline{\mathbb{F}_{q}}\right)$, and let $g: \mathbb{P}^{1} \rightarrow \mathbb{P}^{1}$ be a trigonal map for $S$. If $X$ is the curve formed from $g$ by Recillas' trigonal construction, then there is an isogeny $\phi: J_{H} \rightarrow J_{X}$ defined over $\overline{\mathbb{F}_{q}}$ with kernel $S$.

We will give only a brief description of the geometry of $X$ here, concentrating instead on its explicit construction; we refer the reader to Recillas [12, Donagi 4, $\S 2]$, Birkenhake and Lange [1, §12.7], and Vakil [15] for the geometrical theory (and proofs). The isogeny is analogous to the well-known Richelot isogeny in genus 2 (see Bost and Mestre [2] and Donagi and Livné [5]).

In abstract terms, if $U$ is the subset of the codomain of $g$ above which $g \circ \pi$ is unramified, then $X$ is by definition the closure of the curve over $U$ representing the pushforward to $U$ of the sheaf of sections of $\pi:(g \circ \pi)^{-1}(U) \rightarrow g^{-1}(U)$ (in the étale topology). This means in particular that the $\overline{\mathbb{F}_{q}}$-points of $X$ over an $\overline{\mathbb{F}_{q}}$-point $P$ of $U$ represent partitions of the six $\overline{\mathbb{F}_{q}}$-points of $(g \circ \pi)^{-1}(P)$ into two sets of three exchanged by the hyperelliptic involution. The fibre product of $H$ and $X$ over $\mathbb{P}^{1}$ (with respect to $g \circ \pi$ and $f$ ) is the union of two isomorphic curves, $R$ and $R^{\prime}$, which are exchanged by the involution on $H \times_{\mathbb{P}^{1}} X$ induced by the hyperelliptic involution. The natural projections induce coverings $\pi_{H}: R \rightarrow H$ and $\pi_{X}: R \rightarrow X$ of degrees 2 and 3 , respectively, so $R$ is a $(3,2)$-correspondence between $H$ and $X$. The map $\left(\pi_{X}\right)_{*} \circ\left(\pi_{H}\right)^{*}$ on divisor classes (that is, pulling back from $H$ to $R$, then pushing forward onto $X$ ) induces an isogeny $\phi: J_{H} \rightarrow J_{X}$ with kernel $S 1$ If we replace $R$ with $R^{\prime}$ in the above, we obtain an isogeny isomorphic to $-\phi$. Thus, up to sign, the construction of the isogeny depends only on the subgroup $S$. The curves and morphisms described above form the commutative diagrams shown in Fig. 1,

The hyperelliptic Jacobians form a codimension-1 subspace of the moduli space of 3-dimensional principally polarized abelian varieties. Naïvely, then, if $X$ is a curve of genus 3 selected at random, then the probability that $X$ is hyperelliptic is inversely proportional to $q$; for cryptographically relevant sizes of $q$, this probability should be negligible. This is consistent with our experimental observations. In the sequel, by "a randomly chosen curve $H$ and subgroup $S$ in $\mathcal{S}(H)$ ", we mean a randomly chosen hyperelliptic curve $H$ (in the sense of \$2), together with a subgroup $S$ uniformly randomly chosen from $\mathcal{S}(H)$.

Hypothesis 1 . The probability that the curve $X$ constructed by the trigonal construction for a randomly chosen $H$ and $S$ in $\mathcal{S}(H)$ is hyperelliptic is negligible.

\footnotetext{
$\overline{{ }^{1} \text { Recall that }}\left(\pi_{H}\right)^{*}\left(\sum_{P \in H} n_{P}(P)\right)=\sum_{P \in H} n_{P} \sum_{Q \in \pi_{H}^{-1}(P)}(Q)$, with appropriate multiplicities where $\pi_{H}$ ramifies, and $\left(\pi_{X}\right)_{*}\left(\sum_{Q \in R} m_{Q}(Q)\right)=\sum_{Q \in R} m_{Q}\left(\pi_{X}(Q)\right)$.
} 

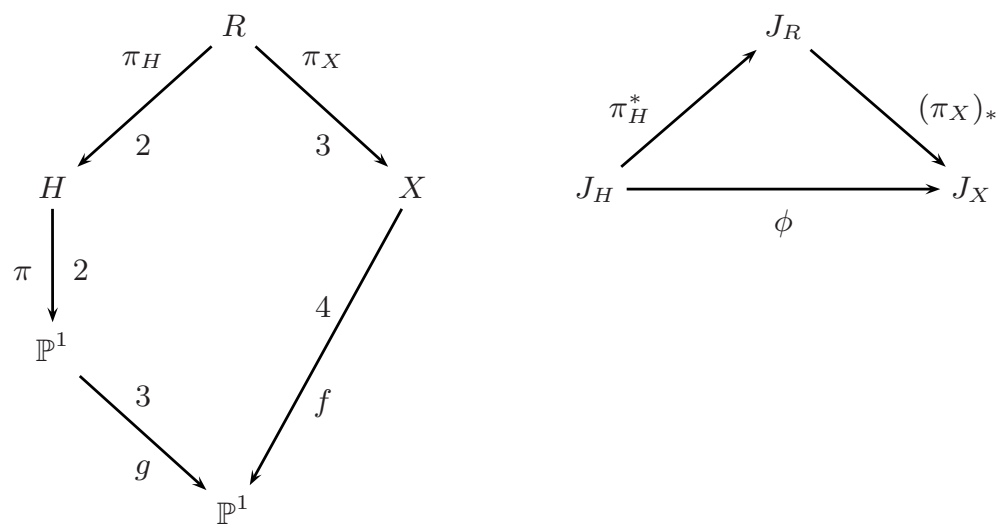

Fig. 1. The curves, Jacobians, and morphisms of $₫$

\section{Computing Trigonal Maps}

Suppose we are given a tractable subgroup $S$ of $J_{H}[2]\left(\overline{\mathbb{F}_{q}}\right)$, corresponding to a partition $\left\{\left\{W_{i}^{\prime}, W_{i}^{\prime \prime}\right\}: 1 \leq i \leq 4\right\}$ of the Weierstrass points of $H$ into pairs. In this section, we compute polynomials $N(x)=x^{3}+a x+b$ and $D(x)=x^{2}+c x+d$ such that the rational map $g: x \mapsto t=N(x) / D(x)$ defines a trigonal map for $S$. Choosing $N$ and $D$ to have degrees 3 and 2 respectively ensures that $g$ maps the point at infinity to the point at infinity; this will be useful to us in 8 .

By definition, $g: \mathbb{P}^{1} \rightarrow \mathbb{P}^{1}$ is a degree-3 map with $g\left(\pi\left(W_{i}^{\prime}\right)\right)=g\left(\pi\left(W_{i}^{\prime \prime}\right)\right)$ for $1 \leq i \leq 4$. We will express $g$ as a composition of maps $g=p \circ e$, where $e: \mathbb{P}^{1} \rightarrow \mathbb{P}^{3}$ is the rational normal embedding defined by

$$
e:(u: v) \longmapsto\left(u_{0}: u_{1}: u_{2}: u_{3}\right)=\left(u^{3}: u^{2} v: u v^{2}: v^{3}\right),
$$

and $p: \mathbb{P}^{3} \rightarrow \mathbb{P}^{1}$ is the projection defined as follows. For each $1 \leq i \leq 4$, we let $L_{i}$ denote the line in $\mathbb{P}^{3}$ passing through $e\left(\pi\left(W_{i}^{\prime}\right)\right)$ and $e\left(\pi\left(W_{i}^{\prime \prime}\right)\right)$. There exists at least one line $L$ intersecting all four of the $L_{i}$ (generically, there are two). We take $p$ to be the projection away from $L$; then $p\left(e\left(\pi\left(W_{i}^{\prime}\right)\right)\right)=p\left(e\left(\pi\left(W_{i}^{\prime \prime}\right)\right)\right)$ for $1 \leq i \leq 4$, so $g=p \circ e$ is a trigonal map for $S$. Given equations for $L$, we can use linear algebra to compute $a, b, c$, and $d$ in $\mathbb{F}_{q}$ such that

$$
L=V\left(u_{0}+a u_{2}+b u_{3}, u_{1}+c u_{2}+d u_{3}\right) .
$$

The projection $p: \mathbb{P}^{3} \rightarrow \mathbb{P}^{1}$ away from $L$ is then defined by

$$
p:\left(u_{0}: u_{1}: u_{2}: u_{3}\right) \longmapsto\left(u_{0}+a u_{2}+b u_{3}: u_{1}+c u_{2}+d u_{3}\right),
$$

and therefore $g=p \circ e$ is defined by

$$
g:(u: v) \longmapsto\left(u^{3}+a u v^{2}+b v^{3}: u^{2} v+c u v^{2}+d v^{3}\right) .
$$


Therefore, if we set $N(x)=x^{3}+a x+b$ and $D(x)=x^{2}+c x+d$, then $g$ will be defined by the rational map $x \longmapsto N(x) / D(x)$.

To compute equations for $L$, we will use the classical theory of Grassmannian varieties (see Griffiths and Harris [7, §1.5] for details). The set of lines in $\mathbb{P}^{3}$ has the structure of an algebraic variety $\operatorname{Gr}(1,3)$, called the Grassmannian. There is a convenient model for $\operatorname{Gr}(1,3)$ as a quadric hypersurface in $\mathbb{P}^{5}$ : if $v_{0}, \ldots, v_{5}$ are coordinates on $\mathbb{P}^{5}$, then we may take

$$
\operatorname{Gr}(1,3):=V\left(v_{0} v_{3}+v_{1} v_{4}+v_{2} v_{5}\right) .
$$

Lemma 2. There is a bijection between points of $\operatorname{Gr}(1,3)\left(\overline{\mathbb{F}_{q}}\right)$ and lines in $\mathbb{P}^{3}$, defined as follows.

1. The point of $\operatorname{Gr}(1,3)\left(\overline{\mathbb{F}_{q}}\right)$ corresponding to the line through $\left(p_{0}: p_{1}: p_{2}: p_{3}\right)$ and $\left(q_{0}: q_{1}: q_{2}: q_{3}\right)$ in $\mathbb{P}^{3}$ has coordinates

$$
\left(\left|\begin{array}{cc}
p_{0} & p_{1} \\
q_{0} & q_{1}
\end{array}\right|:\left|\begin{array}{cc}
p_{0} & p_{2} \\
q_{0} & q_{2}
\end{array}\right|:\left|\begin{array}{cc}
p_{0} & p_{3} \\
q_{0} & q_{3}
\end{array}\right|:\left|\begin{array}{cc}
p_{2} & p_{3} \\
q_{2} & q_{3}
\end{array}\right|:\left|\begin{array}{cc}
p_{3} & p_{1} \\
q_{3} & q_{1}
\end{array}\right|:\left|\begin{array}{cc}
p_{1} & p_{2} \\
q_{1} & q_{2}
\end{array}\right|\right) \text {. }
$$

2. The line in $\mathbb{P}^{3}$ corresponding to a point $\left(\gamma_{0}: \cdots: \gamma_{5}\right)$ of $\operatorname{Gr}(1,3)\left(\overline{\mathbb{F}_{q}}\right)$ is defined by

$$
V\left(\begin{array}{c}
0 u_{0}-\gamma_{3} u_{1}-\gamma_{4} u_{2}-\gamma_{5} u_{3} \\
\gamma_{3} u_{0}+0 u_{1}-\gamma_{2} u_{2}+\gamma_{1} u_{3} \\
\gamma_{4} u_{0}+\gamma_{2} u_{1}+0 u_{2}-\gamma_{0} u_{3} \\
\gamma_{5} u_{0}-\gamma_{1} u_{1}+\gamma_{0} u_{2}+0 u_{3}
\end{array}\right)
$$

(two of the equations will be redundant linear combinations of the others).

Further, if $\left(\gamma_{0}: \cdots: \gamma_{5}\right)$ is a point in $\operatorname{Gr}(1,3)\left(\overline{\mathbb{F}_{q}}\right)$ corresponding to a line $L$, then the points in $\operatorname{Gr}(1,3)\left(\overline{\mathbb{F}_{q}}\right)$ corresponding to lines meeting $L$ are precisely those in the hyperplane defined by $\sum_{i=0}^{5} \gamma_{i} v_{i+3}$, where the subscripts are taken modulo 6 .

Assume that $S$ is represented by a set $\left\{F_{i}=a_{i} u^{2}+b_{i} u v+c_{i} v^{2}: 1 \leq i \leq 4\right\}$ of quadratics, with each $F_{i}$ corresponding to the pair $\left\{W_{i}^{\prime}, W_{i}^{\prime \prime}\right\}$ of Weierstrass points. Elementary calculations show that the point on $\operatorname{Gr}(1,3)$ corresponding to the line $L_{i}$ through $e\left(\pi\left(W_{i}^{\prime}\right)\right)$ and $e\left(\pi\left(W_{i}^{\prime \prime}\right)\right)$ has coordinates

$$
\left(c_{i}^{2}:-c_{i} b_{i}: b_{i}^{2}-a_{i} c_{i}: a_{i}^{2}: a_{i} b_{i}: a_{i} c_{i}\right) .
$$

If $\left(\gamma_{0}: \cdots: \gamma_{5}\right)$ is a point in $\operatorname{Gr}(1,3)\left(\overline{\mathbb{F}_{q}}\right)$ corresponding to a candidate for $L$, then by the second part of Lemma 2 we have $M\left(\gamma_{0}, \ldots, \gamma_{5}\right)^{T}=0$, where

$$
M=\left(\begin{array}{cccccc}
a_{1}^{2} & a_{1} b_{1} & a_{1} c_{1} & c_{1}^{2} & -c_{1} b_{1} & \left(b_{1}^{2}-a_{1} c_{1}\right) \\
a_{2}^{2} & a_{2} b_{2} & a_{2} c_{2} & c_{2}^{2} & -c_{2} b_{2} & \left(b_{2}^{2}-a_{2} c_{2}\right) \\
a_{3}^{2} & a_{3} b_{3} & a_{3} c_{3} & c_{3}^{2} & -c_{3} b_{3} & \left(b_{3}^{2}-a_{3} c_{3}\right) \\
a_{4}^{2} & a_{4} b_{4} & a_{4} c_{4} & c_{4}^{2} & -c_{4} b_{4} & \left(b_{4}^{2}-a_{4} c_{4}\right)
\end{array}\right) .
$$

The kernel of $M$ is two-dimensional, corresponding to a line in $\mathbb{P}^{5}$. Let $\{\underline{\alpha}, \beta\}$ be a basis for $\operatorname{ker} M$, writing $\underline{\alpha}=\left(\alpha_{0}, \ldots, \alpha_{5}\right)$ and $\beta=\left(\beta_{0}, \ldots, \beta_{5}\right)$. If $S$ is $\mathbb{F}_{q^{-}}$ rational, then so is $\operatorname{ker} M$, so we may take the $\alpha_{i}$ and $\beta_{i}$ to be in $\mathbb{F}_{q}$. We want to 
find a point $P_{L}=\left(\alpha_{0}+\lambda \beta_{0}: \cdots: \alpha_{5}+\lambda \beta_{5}\right)$ where the line in $\mathbb{P}^{5}$ corresponding to $\operatorname{ker} M$ intersects with $\operatorname{Gr}(1,3)$. The points $\left(u_{0}: \ldots: u_{3}\right)$ on the line $L$ in $\mathbb{P}^{3}$ corresponding to $P_{L}$ satisfy $\left(M_{\underline{\alpha}}+\lambda M_{\underline{\beta}}\right)\left(u_{0}, \ldots, u_{3}\right)^{T}=0$, where

$$
M_{\underline{\alpha}}:=\left(\begin{array}{rrrr}
0 & -\alpha_{3} & -\alpha_{4} & -\alpha_{5} \\
\alpha_{3} & 0 & -\alpha_{2} & \alpha_{1} \\
\alpha_{4} & \alpha_{2} & 0 & -\alpha_{0} \\
\alpha_{5} & -\alpha_{1} & \alpha_{0} & 0
\end{array}\right) \quad \text { and } \quad M_{\underline{\beta}}:=\left(\begin{array}{rrrr}
0 & -\beta_{3} & -\beta_{4} & -\beta_{5} \\
\beta_{3} & 0 & -\beta_{2} & \beta_{1} \\
\beta_{4} & \beta_{2} & 0 & -\beta_{0} \\
\beta_{5} & -\beta_{1} & \beta_{0} & 0
\end{array}\right) .
$$

By part (2) of Lemma 2, the rank of $M_{\underline{\alpha}}+\lambda M_{\underline{\beta}}$ is 2. Using the expression

$$
\operatorname{det}\left(M_{\underline{\alpha}}+\lambda M_{\underline{\beta}}\right)=\left(\frac{1}{2}\left(\sum_{i=0}^{6} \beta_{i} \beta_{i+3}\right) \lambda^{2}+\left(\sum_{i=0}^{6} \alpha_{i} \beta_{i+3}\right) \lambda+\frac{1}{2} \sum_{i=0}^{6} \alpha_{i} \alpha_{i+3}\right)^{2}
$$

(where the subscripts are taken modulo 6), we see that this occurs precisely when $\operatorname{det}\left(M_{\underline{\alpha}}+\lambda M_{\beta}\right)=0$. We can therefore solve $\operatorname{det}\left(M_{\underline{\alpha}}+\lambda M_{\underline{\beta}}\right)=0$ to determine a value for $\lambda$, and to see that $\mathbb{F}_{q}(\lambda)$ is at most a quadratic extension of $\mathbb{F}_{q}$. Considering the discriminant of $\operatorname{det}\left(M_{\underline{\alpha}}+\lambda M_{\underline{\beta}}\right)$ gives us an explicit criterion for determining whether a given tractable subgroup has a rational trigonal map.

Proposition 2. Suppose $S$ is a subgroup in $\mathcal{S}(H)$, and let $\left\{\underline{\alpha}=\left(\alpha_{i}\right), \beta=\left(\beta_{i}\right)\right\}$ be any $\mathbb{F}_{q}$-rational basis of the nullspace of the matrix $M$ defined in (1). There exists an $\mathbb{F}_{q}$-rational trigonal map for $S$ if and only if

$$
\left(\sum_{i=0}^{6} \alpha_{i} \beta_{i+3}\right)^{2}-\left(\sum_{i=0}^{6} \alpha_{i} \alpha_{i+3}\right)\left(\sum_{i=0}^{6} \beta_{i} \beta_{i+3}\right)
$$

is a square in $\mathbb{F}_{q}$, where the subscripts are taken modulo 6 .

Finally, we use Gaussian elimination to compute $a, b, c$, and $d$ in $\mathbb{F}_{q}(\lambda)$ such that $(1,0, a, b)$ and $(0,1, c, d)$ generate the rowspace of $M_{\underline{\alpha}}+\lambda M_{\underline{\beta}}$. We may then take $L=V\left(u_{0}+a u_{2}+u_{3}, u_{1}+c u_{2}+d u_{3}\right)$. Both $L$ and the projection $p: \mathbb{P}^{3} \rightarrow \mathbb{P}^{1}$ with centre $L$ are defined over $\mathbb{F}_{q}(\lambda)$. Having computed $L$, we compute the projection $p$, the embedding $e$, and the trigonal map $g=p \circ e$ as above.

Proposition 2 shows that the rationality of a trigonal map for a tractable subgroup $S$ depends only upon whether an element of $\mathbb{F}_{q}$ depending on $S$ is a square. It seems reasonable to assume that these field elements are uniformly distributed for random choices of $H$ and $S$, and indeed this is consistent with our experimental observations. Since a uniformly randomly chosen element of $\mathbb{F}_{q}$ is a square with probability $\sim 1 / 2$, we propose the following hypothesis.

Hypothesis 2. The probability that there exists an $\mathbb{F}_{q}$-rational trigonal map for a randomly chosen hyperelliptic curve $H$ over $\mathbb{F}_{q}$ and subgroup $S$ in $\mathcal{S}(H)$ is $1 / 2$.

\section{Equations for the Isogeny}

Suppose we have a tractable subgroup $S$ and a trigonal map $g$ for $S$. We will now perform an explicit trigonal construction on $g$ to compute a curve $X$ and 
an isogeny $\phi: J_{H} \rightarrow J_{X}$ with kernel $S$. We assume that $g$ has been derived as in $\$ 5$, and in particular that $g$ maps the point at infinity to the point at infinity.

Let $U$ be the subset of $\mathbb{A}^{1}=\mathbb{P}^{1} \backslash\{(1: 0)\}$ above which $g \circ \pi$ is unramified. We let $\left.X\right|_{U}$ denote $f^{-1}(U)$, and let $\left.H\right|_{U}$ denote $(g \circ \pi)^{-1}(U)$.

By definition, every point $P$ in $\left.X\right|_{U}\left(\overline{\mathbb{F}_{q}}\right)$ corresponds to a pair of triples of points in $\left.H\right|_{U}\left(\overline{\mathbb{F}_{q}}\right)$, exchanged by the hyperelliptic involution, with each triple supported on the fibre of $g \circ \pi$ over $f(P)$. We will construct a model of the abstract curve $\left.X\right|_{U}$ in $U \times \mathbb{A}^{6}$. We will not prove that our model is isomorphic to the abstract curve, but we will exhibit a bijection of geometric points.

To be more explicit, suppose $Q$ is a generic point of $U$. Since $g \circ \pi$ is unramified above $Q$, we may choose preimages $P_{1}, P_{2}$ and $P_{3}$ of $Q$ such that

$$
(g \circ \pi)^{-1}(Q)=\left\{P_{1}, P_{2}, P_{3}, \iota\left(P_{1}\right), \iota\left(P_{2}\right), \iota\left(P_{3}\right)\right\} .
$$

The four points on $X$ in the preimage $f^{-1}(Q)$ correspond to partitions of the six points in $(g \circ \pi)^{-1}(Q)$ into two unordered triples exchanged by the hyperelliptic involution:

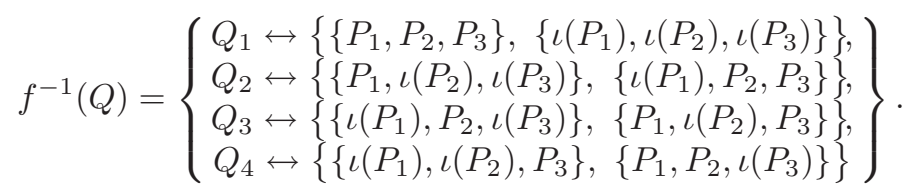

Every triple is cut out by an ideal $(a(x), y-b(x))$, where $a$ is a cubic polynomial, $b$ is a quadratic polynomial, and $b^{2} \equiv F(\bmod a)$. If we require $a$ to be monic, then there is a one-to-one correspondence between such ideals and triples; this is the well-known Mumford representation. The triple is defined over $\mathbb{F}_{q}$ if and only if $a$ and $b$ are defined over $\mathbb{F}_{q}$. For example, the triple $\left\{P_{1}, P_{2}, P_{3}\right\}$ corresponds to the ideal $(a(x), y-b(x))$ where $a(x)=\prod_{i}\left(x-x\left(P_{i}\right)\right)$ and $b$ satisfies $y\left(P_{i}\right)=b\left(x\left(P_{i}\right)\right)$ for $1 \leq i \leq 3$; the Lagrange interpolation formula may be used to compute $b$. If $(a(x), y-b(x))$ corresponds to one triple in a partition, then $(a(x), y+b(x))$ corresponds to the other triple. The union of the triples equals the whole fibre $(g \circ \pi)^{-1}(Q)$, and since the union of the triples is cut out by the product of the corresponding ideals, we know that $a(x)$ must cut out the fibre of $g \circ \pi$ over $Q$. Therefore, we have $a(x)=N(x)-t(Q) D(x)$.

For notational convenience, we define

$$
G(t, x)=x^{3}+g_{2}(t) x^{2}+g_{1}(t) x+g_{0}(t):=N(x)-t D(x) .
$$

Let $f_{0}, f_{1}$, and $f_{2}$ be the elements of $\mathbb{F}_{q}[t]$ such that

$$
f_{0}(t)+f_{1}(t) x+f_{2}(t) x^{2} \equiv F(x) \quad(\bmod G(t, x)) .
$$

The triples in the pairs over the generic point of $U$ have Mumford representatives of the form $\left(G(t, x), y-\left(b_{0}+b_{1} x+b_{2} x^{2}\right)\right)$, where

$$
\left(b_{0}+b_{1} x+b_{2} x^{2}\right)^{2} \equiv F(x) \quad(\bmod G(t, x)) .
$$


Viewing $b_{0}, b_{1}$, and $b_{2}$ as coordinates on $\mathbb{A}^{3}$, we expand both sides of (5) modulo $G(t, x)$ and equate coefficients to obtain a variety $\widetilde{X}$ in $U \times \mathbb{A}^{3}$ parametrizing triples:

$$
\widetilde{X}:=V\left(c_{0}\left(t, b_{0}, b_{1}, b_{2}\right), c_{1}\left(t, b_{0}, b_{1}, b_{2}\right), c_{2}\left(t, b_{0}, b_{1}, b_{2}\right)\right),
$$

where

$$
\begin{aligned}
& c_{0}\left(t, b_{0}, b_{1}, b_{2}\right)=g_{2}(t) g_{0}(t) b_{2}^{2}-2 g_{0}(t) b_{2} b_{1}+b_{0}^{2}-f_{0}(t), \\
& c_{1}\left(t, b_{0}, b_{1}, b_{2}\right)=\left(g_{2}(t) g_{1}(t)-g_{0}(t)\right) b_{2}^{2}-2 g_{1}(t) b_{2} b_{1}+2 b_{1} b_{0}-f_{1}(t), \text { and } \\
& c_{2}\left(t, b_{0}, b_{1}, b_{2}\right)=\left(g_{2}(t)^{2}-g_{1}(t)\right) b_{2}^{2}-2 g_{2}(t) b_{2} b_{1}+2 b_{2} b_{0}+b_{1}^{2}-f_{2}(t) .
\end{aligned}
$$

The Mumford representatives corresponding to the triples in each pair are exchanged by the involution $\iota_{*}: \widetilde{X} \longrightarrow \widetilde{X}$ defined by

$$
\iota_{*}:\left(t, b_{0}, b_{1}, b_{2}\right) \longmapsto\left(t,-b_{0},-b_{1},-b_{2}\right) ;
$$

the curve $\left.X\right|_{U}$ is therefore the quotient of $\tilde{X}$ by the involution $\iota_{*}$. To form this quotient, let $m: U \times \mathbb{A}^{3} \longrightarrow U \times \mathbb{A}^{6}$ be the map defined by

$$
m:\left(t, b_{0}, b_{1}, b_{2}\right) \longmapsto\left(t, b_{00}, b_{01}, b_{02}, b_{11}, b_{12}, b_{22}\right)=\left(t, b_{0}^{2}, b_{0} b_{1}, b_{0} b_{2}, b_{1}^{2}, b_{1} b_{2}, b_{2}^{2}\right) ;
$$

the image $B$ of $m$ is the variety defined by

$$
B=V\left(\begin{array}{c}
b_{01}^{2}-b_{00} b_{11}, b_{01} b_{02}-b_{00} b_{12}, b_{02}^{2}-b_{00} b_{22}, \\
b_{02} b_{11}-b_{01} b_{12}, b_{02} b_{12}-b_{01} b_{22}, b_{12}^{2}-b_{11} b_{22}
\end{array}\right) \subset U \times \mathbb{A}^{3} .
$$

We have $\left.X\right|_{U}=m(\widetilde{X})$, so

$$
\left.X\right|_{U}=V\left(\begin{array}{c}
g_{2} g_{0} b_{22}-2 g_{0} b_{12}+b_{00}-f_{0}, \\
\left(g_{2} g_{1}-g_{0}\right) b_{22}-2 g_{1} b_{12}+2 b_{01}-f_{1}, \\
\left(g_{2}^{2}-g_{1}\right) b_{22}-2 g_{2} b_{12}+2 b_{02}+b_{11}-f_{2}
\end{array}\right) \cap B \subset U \times \mathbb{A}^{6} .
$$

Consider again the fibre of $f: X \rightarrow \mathbb{P}^{1}$ over the generic point $Q=(t)$ of $U$ (as in (4)). If $\left\{P_{1}, P_{2}, P_{3}\right\}$ is one of the triples in a pair in the fibre, then by the Lagrange interpolation formula the value of $b_{2}$ at the corresponding point of $\widetilde{X}$ is

$$
b_{2}=\sum y\left(P_{i}\right) /\left(\left(x\left(P_{i}\right)-x\left(P_{j}\right)\right)\left(x\left(P_{i}\right)-x\left(P_{k}\right)\right)\right),
$$

where the sum is taken over the cyclic permutations $(i, j, k)$ of $(1,2,3)$. Interpolating for all triples in the pairs in the fibre, an elementary but involved symbolic calculation shows that if we define $\Delta_{1}, \Delta_{2}$, and $\Delta_{3}$ by

$$
\Delta_{i}:=\left(x\left(P_{j}\right)-x\left(P_{k}\right)\right)^{2}
$$

and $\Gamma_{1}, \Gamma_{2}$, and $\Gamma_{3}$ by

$$
\Gamma_{i}:=\left(f_{2}(t) x\left(P_{i}\right)^{2}+f_{1}(t) x\left(P_{i}\right)+f_{0}(t)\right) \Delta_{i}=F\left(x\left(P_{i}\right)\right) \Delta_{i}
$$

for each cyclic permutation $(i, j, k)$ of $(1,2,3)$, and set

$$
\Delta:=\Delta_{1} \Delta_{2} \Delta_{3},
$$

then $b_{2}$ satisfies 


$$
\left(\Delta b_{2}^{4}-2\left(\sum_{i} \Gamma_{i}\right) b_{2}^{2}+\frac{1}{\Delta}\left(2\left(\sum_{i} \Gamma_{i}^{2}\right)-\left(\sum_{i} \Gamma_{i}\right)^{2}\right)\right)^{2}-64\left(\prod_{i} \Gamma_{i}\right) b_{2}^{2}=0 .
$$

Now $\Delta, \sum_{i} \Gamma_{i}, \sum_{i} \Gamma_{i}^{2}$, and $\prod_{i} \Gamma_{i}$ are symmetric functions with respect to permutations of the points in the fibre $g^{-1}(Q)=g^{-1}((t))$. They are therefore polynomials in the homogeneous elementary symmetric functions

$$
e_{1}=\sum x\left(P_{i}\right), \quad e_{2}=\sum x\left(P_{i}\right) x\left(P_{j}\right), \quad \text { and } \quad e_{3}=\prod x\left(P_{i}\right),
$$

which are polynomials in $t$. Indeed, the $e_{i}$ are given by the coefficients of $G(t, x)$ :

$$
e_{1}=-g_{2}(t), \quad e_{2}=g_{1}(t), \quad \text { and } \quad e_{3}=-g_{0}(t) .
$$

Expressing $\Delta, \sum_{i} \Gamma_{i}, \sum_{i} \Gamma_{i}^{2}$, and $\prod_{i} \Gamma_{i}$ in terms of $f_{0}, f_{1}, f_{2}, g_{0}, g_{1}$, and $g_{2}$, and then simplifying, we define $\delta_{4}, \delta_{2}$, and $\delta_{0}$ by

$$
\begin{aligned}
& \delta_{4}:=-27 g_{0}^{2}+18 g_{0} g_{1} g_{2}-4 g_{0} g_{2}^{3}-4 g_{1}^{3}+g_{1}^{2} g_{2}^{2}, \\
& \delta_{2}:=12 f_{0} g_{1}-4 f_{0} g_{2}^{2}-18 f_{1} g_{0}+2 f_{1} g_{1} g_{2}+12 f_{2} g_{0} g_{2}-4 f_{2} g_{1}^{2}, \\
& \delta_{0}:=-4 f_{0} f_{2}+f_{1}^{2},
\end{aligned}
$$

and $s$ by

$$
\begin{aligned}
s:= & f_{0}^{3}-f_{0}^{2} f_{1} g_{2}-2 f_{0}^{2} f_{2} g_{1}+f_{0}^{2} f_{2} g_{2}^{2}+f_{0} f_{1}^{2} g_{1}+3 f_{0} f_{1} f_{2} g_{0}-f_{0} f_{1} f_{2} g_{1} g_{2} \\
& -2 f_{0} f_{2}^{2} g_{0} g_{2}+f_{0} f_{2}^{2} g_{1}^{2}-f_{1}^{3} g_{0}+f_{1}^{2} f_{2} g_{0} g_{2}-f_{1} f_{2}^{2} g_{0} g_{1}+f_{2}^{3} g_{0}^{2} .
\end{aligned}
$$

Since $s(t)=F\left(x\left(P_{1}\right)\right) F\left(x\left(P_{2}\right)\right) F\left(x\left(P_{3}\right)\right)=\left(y\left(P_{1}\right) y\left(P_{2}\right) y\left(P_{3}\right)\right)^{2}$, there is a square root of $s(t)$ in $\overline{\mathbb{F}_{q}}[t]$; in fact, it is defined over $\mathbb{F}_{q}(\sqrt{s(0)})$. We therefore define

$$
\delta_{1}:=8 \sqrt{s} .
$$

With this notation (8) becomes $\left(\delta_{4}(t) b_{2}^{4}+\delta_{2}(t) b_{2}^{2}+\delta_{0}(t)\right)^{2}-\delta_{1}(t)^{2} b_{2}^{2}=0$, and hence on $\left.X\right|_{U}$ we have

$$
\left(\delta_{4}(t) b_{22}^{2}+\delta_{2}(t) b_{22}+\delta_{0}(t)\right)^{2}-\delta_{1}(t)^{2} b_{22}=0 .
$$

Observe that (11) gives us a (singular) affine plane model for $X$. We can also use (11) to compute a square root for $b_{22}$ on $\left.X\right|_{U}$ : we have

$$
b_{22}=\rho^{2}, \quad \text { where } \quad \rho:=\frac{\delta_{4}(t) b_{22}^{2}+\delta_{2}(t) b_{22}+\delta_{0}(t)}{\delta_{1}(t)} .
$$

Given a point $\left(t, b_{00}, \ldots, b_{22}\right)$ of $\left.X\right|_{U}$, the two triples of points corresponding to the two points of $\widetilde{X}$ over $\left(t, b_{00}, \ldots, b_{22}\right)$ have Mumford representatives

$$
\left(G(t, x), y-\left(\frac{b_{02}}{\rho}+\frac{b_{12}}{\rho} x+\frac{b_{22}}{\rho} x^{2}\right)\right) \text { and }\left(G(t, x), y+\left(\frac{b_{02}}{\rho}+\frac{b_{12}}{\rho} x+\frac{b_{22}}{\rho} x^{2}\right)\right) .
$$

We will now compute the Recillas correspondence $R$ inducing the isogeny from $J_{H}$ to $J_{X}$. We know that $R$ is a component of the fibre product $H \times_{\mathbb{P}^{1}} X$ 
(with respect to $g \circ \pi$ and $f$ ). We may realise the open affine subset $\left.H\right|_{U} \times\left._{U} X\right|_{U}$ as the subvariety $V(G(t, x))$ of $\left.H\right|_{U} \times\left. X\right|_{U}$. Now, $V(G(t, x))$ decomposes into two components: clearing denominators in (13), we find $V(G(t, x))=R \cup R^{\prime}$, where

$$
R=V\left(G(t, x),\left(\delta_{4}(t) b_{22}^{2}+\delta_{2}(t) b_{22}+\delta_{0}(t)\right) y-\delta_{1}(t)\left(b_{02}+b_{12} x+b_{22} x^{2}\right)\right)
$$

and

$$
R^{\prime}=V\left(G(t, x),\left(\delta_{4}(t) b_{22}^{2}+\delta_{2}(t) b_{22}+\delta_{0}(t)\right) y+\delta_{1}(t)\left(b_{02}+b_{12} x+b_{22} x^{2}\right)\right) .
$$

The natural projections $\pi_{X}: R \rightarrow X$ and $\pi_{H}: R \rightarrow H$ send $\left(x, y, t, b_{00}, \ldots, b_{22}\right)$ to $\left(t, b_{00}, \ldots, b_{22}\right)$ and $(x, y)$, respectively. On the level of divisor classes, the isogeny $\phi: J_{H} \rightarrow J_{X}$ is made explicit by the map

$$
\phi=\left(\pi_{X}\right)_{*} \circ\left(\pi_{H}\right)^{*} .
$$

In terms of ideals cutting out effective divisors, $\phi$ is realized by the map

$$
I_{D} \longmapsto\left(I_{D}+\left(G(t, x), y-\left(\frac{b_{02}}{\rho}+\frac{b_{12}}{\rho} x+\frac{b_{22}}{\rho} x^{2}\right)\right)\right) \cap \mathbb{F}_{q}\left[s, t, b_{00}, \ldots, b_{22}\right] .
$$

Taking $R^{\prime}$ in place of $R$ in the above gives an isogeny equal to $-\phi$.

It remains to determine the rationality of the isogeny. We see from (7) that $X$ is defined over the field of definition of $g$. The correspondence $R$, and the isogeny $\phi$, are both defined over the field of definition of $\rho$, which is $\mathbb{F}_{q}(\sqrt{s(0)})$. This gives us a useful criterion for when an $\mathbb{F}_{q}$-rational subgroup $S$ and trigonal map $g$ lead to an $\mathbb{F}_{q}$-rational isogeny.

Proposition 3. If $S$ is a subgroup in $\mathcal{S}(H)$ with an $\mathbb{F}_{q}$-rational trigonal map $g$, then the trigonal construction on $g$ yields an $\mathbb{F}_{q}$-rational isogeny if and only if $s(0)$ is a square in $\mathbb{F}_{q}$, where $s$ is defined in (9).

Remark 4. If $\phi$ is not $\mathbb{F}_{q}$-rational, then $J_{X}$ is a quadratic twist of $J_{H} / S$ (see 99 ).

If we assume that the values $s(0)$ are uniformly distributed for randomly chosen $H, S$, and $g$, then the probability that $s(0)$ is a square in $\mathbb{F}_{q}$ is $1 / 2$. Indeed, it is easily seen that $s(0)$ is a square for $H$ if and only if it is not a square for the quadratic twist of $H$. This suggests that the probability that we can compute an $\mathbb{F}_{q}$-rational $\phi$ given an $\mathbb{F}_{q}$-rational $g$ for a randomly chosen $H$ and $S$ in $\mathcal{S}(H)$ is $1 / 2$. This is consistent with our experimental observations, so we propose Hypothesis 3 .

Hypothesis 3. Given a randomly chosen hyperelliptic curve $H$ over $\mathbb{F}_{q}$ and tractable subgroup $S$ in $\mathcal{S}(H)$ with an $\mathbb{F}_{q}$-rational trigonal map $g$, the probability that we can compute an $\mathbb{F}_{q}$-rational isogeny $\phi$ with kernel $S$ is $1 / 2$. 


\section{Computing Isogenies}

Suppose we are given a hyperelliptic curve $H$ of genus 3 , defined over $\mathbb{F}_{q}$, and a DLP in $J_{H}\left(\mathbb{F}_{q}\right)$ to solve. Our goal is to compute a nonsingular plane quartic curve $C$ and an isogeny $J_{H} \rightarrow J_{C}$ so that we can reduce to a DLP in $J_{C}\left(\mathbb{F}_{q}\right)$.

We begin by computing the set $\mathcal{S}(H)$ of $\mathbb{F}_{q}$-rational tractable subgroups of $J_{H}[2]\left(\overline{\mathbb{F}_{q}}\right)$. For each $S$ in $\mathcal{S}(H)$, we apply Proposition 2 to determine whether there exists an $\mathbb{F}_{q}$-rational trigonal map $g$ for $S$. If so, we use the formulae of 95 to compute $g$; if not, we move on to the next $S$. Having computed $g$, we apply Proposition 3 to determine whether we can compute an isogeny over $\mathbb{F}_{q}$. If so, we use the formulae of $\sqrt{6}$ to compute equations for $X$ and the isogeny $J_{H} \rightarrow J_{X}$; if not, we move on to the next $S$.

The formulae of 96 give an affine model of $X$ in $\mathbb{A}^{1} \times \mathbb{A}^{6}$. In order to apply Diem's algorithm to the DLP in $J_{X}$, we need a nonsingular plane quartic model of $X$ : that is, a nonsingular curve $C \subset \mathbb{P}^{2}$ isomorphic to $X$, cut out by a quartic form. Such a model exists if and only if $X$ is not hyperelliptic. To find $C$, we compute a basis $\mathcal{B}$ of the Riemann-Roch space of a canonical divisor of $X$. This is a routine geometrical calculation; some of the various approaches are listed in Hess 8]. In practice, the algorithms implemented in Magma 9] compute $\mathcal{B}$ very quickly. The three functions in $\mathcal{B}$ define a map $\psi: X \rightarrow \mathbb{P}^{2}$. If the image of $\psi$ is a conic, then $X$ is hyperelliptic; in this situation, we move on to the next $S$. Otherwise, the image of $\psi$ is a nonsingular plane quartic $C$, and $\psi$ restricts to an isomorphism $\psi: X \rightarrow C$.

If the procedure outlined above succeeds for some $S$ in $\mathcal{S}(H)$, then we have computed an explicit $\mathbb{F}_{q}$-rational isogeny $\psi_{*} \circ \phi: J_{H} \rightarrow J_{C}$. We can then map our DLP from $J_{H}\left(\mathbb{F}_{q}\right)$ into $J_{C}\left(\mathbb{F}_{q}\right)$, and solve using Diem's algorithm.

We emphasize that the entire procedure is very fast: as we saw above, the curve $X$ and the isogeny can be constructed using only low-degree polynomial arithmetic and low-dimensional linear algebra. For a rough idea of the computational effort involved, given a random $H$ over a 160-bit prime field, a naïve implementation of our algorithms in Magma 9. computes the trigonal map $g$, the curve $X$, the nonsingular plane quartic $C$, and the isogeny $\phi: J_{H} \rightarrow J_{C}$ in a few seconds on a $1.2 \mathrm{GHz}$ laptop. Since the difficulty of the construction depends only upon the size of $\mathbb{F}_{q}$ (and not upon the size of the DLP subgroup of $J_{H}\left(\mathbb{F}_{q}\right)$ ), we may conclude that instances of the DLP in 160-bit Jacobians chosen for cryptography may also be reduced to instances of the DLP in nonhyperelliptic Jacobians in a mattter of seconds.

Example 1. We will give an example over a small field. Let $H$ be the hyperelliptic curve over $\mathbb{F}_{37}$ defined by

$$
H: y^{2}=x^{7}+28 x^{6}+15 x^{5}+20 x^{4}+33 x^{3}+12 x^{2}+29 x+2 .
$$

Using the ideas in 93 , we see that $J_{H}$ has one $\mathbb{F}_{37}$-rational tractable subgroup:

$$
\mathcal{S}(H)=\{S\} \quad \text { where } \quad S=\left\{\begin{array}{l}
u^{2}+\xi_{1} u v+\xi_{2} v^{2}, u^{2}+\xi_{1}^{37} u v+\xi_{2}^{37} v^{2}, \\
u^{2}+\xi_{1}^{37^{2}} u v+\xi_{2}^{37^{2}} v^{2}, u v+20 v^{2}
\end{array}\right\},
$$


where $\xi_{1}$ is an element of $\mathbb{F}_{37^{3}}$ satisfying $\xi_{1}^{3}+29 \xi_{1}^{2}+9 \xi_{1}+13=0$, and $\xi_{2}=\xi_{1}^{50100}$. Applying the methods of $\$ 5$, we compute polynomials

$$
N(x)=x^{3}+16 x+22 \text { and } D(x)=x^{2}+32 x+18
$$

such that $g: x \longmapsto N(x) / D(x)$ is an $\mathbb{F}_{37}$-rational trigonal map for $S$. Using the formulae of $\$ 6$, we compute a curve $X \subset \mathbb{A}^{1} \times \mathbb{A}^{6}$ of genus 3 , defined by

$$
X=V\left(\begin{array}{l}
19 t^{5}+10 t^{4}+12 t^{3}+18 t^{2} b_{22}+7 t^{2}+36 t b_{12}+15 t b_{22}+t+b_{00}+30 b_{12}+30, \\
5 t^{5}+26 t^{4}+15 t^{3}+32 t^{2} b_{22}+23 t^{2}+27 t b_{12}+2 t b_{22}+19 t+2 b_{01}+5 b_{12}+15 b_{22}+17, \\
36 t^{5}+29 t^{4}+7 t^{3}+t^{2} b_{22}+13 t^{2}+2 t b_{12}+32 t b_{22}+21 t+2 b_{02}+b_{11}+21 b_{22}+18, \\
b_{00} b_{11}-b_{01}^{2}, b_{00} b_{12}-b_{01} b_{02}, b_{00} b_{22}-b_{02}^{2}, b_{02} b_{11}-b_{01} b_{12}, b_{02} b_{12}-b_{01} b_{22}, b_{12}^{2}-b_{11} b_{22}
\end{array}\right)
$$

together with a map on divisors inducing an isogeny from $J_{H}$ to $J_{X}$ with kernel $S$ (we will not show the equations, for lack of space). Computing the canonical morphism of $X$, we find that $X$ is non-hyperelliptic, and isomorphic to the nonsingular plane quartic curve

$$
C=V\left(\begin{array}{c}
u^{4}+26 u^{3} v+2 u^{3} w+17 u^{2} v^{2}+9 u^{2} v w+20 u^{2} w^{2}+34 u v^{3}+24 u v^{2} w \\
+5 u v w^{2}+36 u w^{3}+19 v^{4}+13 v^{3} w+v^{2} w^{2}+23 v w^{3}+5 w^{4}
\end{array}\right) .
$$

Composing the isomorphism with the isogeny $J_{H} \rightarrow J_{X}$, we obtain an explicit isogeny $\phi: J_{H} \rightarrow J_{C}$. Using Magma, we can verify that $J_{H}$ and $J_{C}$ are isogenous by checking that the zeta functions of $H$ and $C$ are identical: indeed,

$$
Z(H ; T)=Z(C ; T)=\frac{37^{3} T^{6}+4 \cdot 37^{2} T^{5}-6 \cdot 37 T^{4}-240 T^{3}-6 T^{2}+4 T+1}{37 T^{2}-38 T+1} .
$$

If $D$ and $D^{\prime}$ are the divisor classes on $H$ with Mumford representatives $\left(x^{2}+13 x+\right.$ $29, y-10 x-2)$ and $\left(x^{2}+19 x+18, y-15 x-2\right)$, respectively, then $D^{\prime}=[22359] D$. Applying $\phi$, we find that

$$
\begin{aligned}
\phi(D) & =[(7: 18: 1)+(34: 34: 1)-(18: 22: 1)-(15: 33: 1)] \quad \text { and } \\
\phi\left(D^{\prime}\right) & =[(7: 23: 1)+(6: 13: 1)-(13: 15: 1)-(7: 18: 1)] ;
\end{aligned}
$$

direct calculation verifies that $\phi\left(D^{\prime}\right)=[22359] \phi(D)$, as expected.

\section{Expectation of Existence of Computable Isogenies}

We conclude by estimating the proportion of genus 3 hyperelliptic Jacobians over $\mathbb{F}_{q}$ for which the methods of this article produce a rational isogeny - and thus the proportion of hyperelliptic curves for which the DLP may be solved using Diem's algorithm - as $q$ tends to infinity. We will assume that if we are given a selection of $\mathbb{F}_{q}$-rational tractable subgroups, then it is equally probable that any one of them will yield a rational isogeny. This appears consistent with our experimental observations.

Hypothesis 4. If $S_{1}$ and $S_{2}$ are distinct subgroups in $\mathcal{S}(H)$, then the probability that we can compute an $\mathbb{F}_{q}$-rational isogeny with kernel $S_{1}$ is independent of the probability that we can compute an $\mathbb{F}_{q}$-rational isogeny with kernel $S_{2}$. 
Theorem 2. Assume Hypotheses 1, 2, 3, and 4. Let $\mathcal{T}$ be the set of integer partitions of 8; for each $T$ in $\mathcal{T}$ we define $\nu_{T}(n)$ to be the multiplicity of $n$ in $T$, and define $s(T)=\# \mathcal{S}(H)$, where $H$ is any hyperelliptic curve over $\mathbb{F}_{q}$ such that the multiset of degrees of the $\mathbb{F}_{q}$-irreducible factors of its hyperelliptic polynomial coincides with T. As q tends to infinity, the expectation that the algorithms in this article will give a reduction of the DLP in a subgroup of $J_{H}\left(\mathbb{F}_{q}\right)$ for a randomly chosen hyperelliptic curve $H$ of genus 3 over $\mathbb{F}_{q}$ to a subgroup of $J_{C}\left(\mathbb{F}_{q}\right)$ for some nonsingular plane quartic curve $C$ is

$$
\sum_{T \in \mathcal{T}}\left(\left(1-(1-1 / 4)^{s(T)}\right) / \prod_{n \in T}\left(\nu_{T}(n) ! \cdot n^{\nu_{T}(n)}\right)\right) \approx 0.1857 .
$$

Proof. Hypotheses 1, 2, 3, and 4, together imply that if $H$ is a randomly chosen hyperelliptic curve of genus 3 over $\mathbb{F}_{q}$, then the probability that we will succeed in computing a rational isogeny from $J_{H}$ is

$$
1-(1-(1 / 2 \cdot 1 / 2))^{\# \mathcal{S}(H)} .
$$

Lemma 1 implies that $\mathcal{S}(H)$ depends only on the degrees of the irreducible factors of $\widetilde{F}$. For each $T$ in $\mathcal{T}$, let $N_{q}(T)$ denote the number of homogeneous squarefree polynomials over $\mathbb{F}_{q}$ whose multiset of degrees of irreducible factors coincides with $T$. By (15), the expectation that we can compute an $\mathbb{F}_{q}$-rational isogeny from the Jacobian of a randomly chosen hyperelliptic curve to the Jacobian of a non-hyperelliptic curve using the methods in this article is

$$
E_{q}:=\frac{\sum_{T \in \mathcal{T}}\left(1-(1-1 / 4)^{s(T)}\right) N_{q}(T)}{\sum_{T \in \mathcal{T}} N_{q}(T)} .
$$

Let $N_{q}(n)$ denote the number of monic irreducible polynomials of degree $n$ over $\mathbb{F}_{q}$; clearly $N_{q}(T)=(q-1) \prod_{n \in T}\left(\begin{array}{l}N_{q}(n) \\ \nu_{T}(n)\end{array}\right)$. Computing $N_{q}(T)$ is a straightforward combinatorial exercise: we find that $N_{q}(n)=q^{n} / n+O\left(q^{n-1}\right)$, so

$$
N_{q}(T)=\left(\prod_{n \in T}\left(\nu_{T}(n) ! \cdot n^{\nu_{T}(n)}\right)^{-1}\right) q^{9}+O\left(q^{8}\right),
$$

and $\sum_{T \in \mathcal{T}} N_{q}(T)=q^{9}+O\left(q^{8}\right)$. Therefore, as $q$ tends to infinity, we have

$$
\lim _{q \rightarrow \infty} E_{q}=\sum_{T \in \mathcal{T}}\left(\left(1-(1-1 / 4)^{s(T)}\right) / \prod_{n \in T}\left(\nu_{T}(n) ! \cdot n^{\nu_{T}(n)}\right)\right) .
$$

The result follows upon explicitly computing this sum using the values for $s(T)$ derived in Lemma 1

Theorem 2 gives the expectation that we can construct an explicit isogeny for a randomly selected hyperelliptic curve. However, looking at the table in Lemma 1 . we see that we can ensure that a particular curve has no rational isogenies if its hyperelliptic polynomial has an irreducible factor of degree 5 or 7 (or a single 
irreducible factor of degree 3). It may be difficult to efficiently construct a curve in this form if we are using the CM construction, for example, to ensure that the Jacobian has a large prime-order subgroup. In any case, it is interesting to note that the security of genus 3 hyperelliptic Jacobians depends significantly upon the factorization of their hyperelliptic polynomials. This observation has no analogue for elliptic curves or Jacobians of genus 2 curves.

Remark 5. We noted in 4 that the isomorphism class of the curve $X$ in the trigonal construction is independent of the choice of trigonal map. If there is no rational trigonal map for a given subgroup $S$, then the methods of $\$ 5$ construct a pair of Galois-conjugate trigonal maps $g_{1}$ and $g_{2}$ (corresponding to the roots of (2) instead. Applying the trigonal construction to $g_{1}$ and $g_{2}$, we obtain a pair of curves $X_{1}$ and $X_{2}$ over $\mathbb{F}_{q^{2}}$, which must be twists. If the isomorphism between these two curves was made explicit, then Galois descent could be used to compute a curve $X$ in their isomorphism class defined over $\mathbb{F}_{q}$, and hence a nonsingular plane quartic $C$ and isogeny $J_{H} \rightarrow J_{C}$ over $\mathbb{F}_{q}$. This approach would allow us to replace the $1 / 4$ in (15) and (14) with $1 / 2$, raising the expectation of success in Theorem 2 to over $30 \%$.

\section{$9 \quad$ Other Isogenies}

In this article, we have used a special kind of $(2,2,2)$-isogeny for moving instances of the DLP from hyperelliptic to non-hyperelliptic Jacobians. More generally, we can consider using other types of isogenies. There are two important issues to consider here: the first is a theoretical restriction on the types of subgroups $S$ of $J_{H}$ that can be kernels of isogenies of Jacobians, and the second is a practical restriction on the isogenies that we can currently compute.

Suppose $J_{H}$ is a hyperelliptic Jacobian, and $S$ a (finite) $\mathbb{F}_{q}$-rational subgroup of $J_{H}$. The quotient $J_{H} \rightarrow J_{H} / S$ exists as an isogeny of abelian varieties (see Serre [14, §III.3.12], for example). For the quotient to be an isogeny of Jacobians, there must be an integer $m$ such that $S$ is a maximal isotropic subgroup with respect to the $m$-Weil pairing (see Proposition 16.8 of Milne [10]): this ensures that the canonical polarization on $J_{H}$ induces a principal polarization on the quotient. The simplest such subgroups have the form $(\mathbb{Z} / l \mathbb{Z})^{3}$ where $l$ is prime. The theorem of Oort and Ueno [1] then guarantees that there will be an isomorphism over $\overline{\mathbb{F}}_{q}$ from $J_{H} / S$ to the Jacobian $J_{X}$ of some (possibly reducible) curve $X$. Standard arguments from Galois cohomology (see Serre [13, §III.1], for example) show that the isomorphism is defined over either $\mathbb{F}_{q}$ or $\mathbb{F}_{q^{2}}$, so $J_{H} / S$ is either isomorphic to $J_{X}$ over $\mathbb{F}_{q}$ or a quadratic twist of $J_{X}$. We can expect $X$ to be isomorphic to a non-hyperelliptic curve $C$. To compute an $\mathbb{F}_{q}$-rational isogeny from $J_{H}$ to a non-hyperelliptic Jacobian, therefore, the minimum requirement is an $\mathbb{F}_{q}$-rational $l$-Weil isotropic subgroup of $J_{H}\left(\overline{\mathbb{F}_{q}}\right)$ isomorphic to $(\mathbb{Z} / l \mathbb{Z})^{3}$ for some prime $l$.

The second and more serious problem is the lack of general constructions for isogenies in genus 3. Apart from integer and Frobenius endomorphisms, we know of no constructions for explicit isogenies of general Jacobians of genus 3 
hyperelliptic curves other than the one presented here. This situation stands in marked contrast to the case of isogenies of elliptic curves, which have been made completely explicit by Vélu [16. Deriving general formulae for explicit isogenies in genus 3 (and 2) remains a significant problem in computational number theory.

\section{Acknowledgements}

This work was supported by EPSRC grant EP/C014839/1, and a large part of it was completed in the Department of Mathematics at Royal Holloway, University of London. The author gratefully acknowledges Roger Oyono and Christophe Ritzenthaler for discussions which inspired this research, Steven Galbraith for his advice, and the (anonymous) referees for their suggestions.

\section{References}

1. Birkenhake, C., Lange, H.: Complex abelian varieties (2e). In: Grundlehren der mathematischen Wissenschaften, vol. 302, Springer, Heidelberg (2004)

2. Bost, J.-B., Mestre, J.-F.: Moyenne arithmético-géométrique et périodes des courbes de genre 1 et 2. Gaz. Math. Soc. France 38, 36-64 (1988)

3. Diem, C.: An index calculus algorithm for plane curves of small degree. In: Hess, F., Pauli, S., Pohst, M. (eds.) ANTS 2006. LNCS, vol. 4076, Springer, Heidelberg (2006)

4. Donagi, R.: The fibres of the Prym map. Curves, Jacobians, and abelian varieties (Amherst, MA, 1990), Contemp. Math. 136, 55-125 (1992)

5. Donagi, R., Livné, R.: The arithmetic-geometric mean and isogenies for curves of higher genus. Ann. Scuola Norm. Sup. Pisa Cl. Sci(4) 28(2), 323-339 (1999)

6. Gaudry, P., Thomé, E., Thériault, N., Diem, C.: A double large prime variation for small genus hyperelliptic index calculus. Math. Comp. 76, 475-492 (2007)

7. Griffiths, P., Harris, J.: Principles of Algebraic Geometry. Wiley and Sons, Chichester (1978)

8. Hess, F.: Computing Riemann-Roch spaces in algebraic function fields and related topics. J. Symbolic Computation 33(4), 425-445 (2002)

9. The Magma computational algebra system, http://magma.maths.usyd.edu.au/

10. Milne, J.S.: Abelian varieties. In: Arithmetic geometry (Storrs, Conn., 1984), pp. 103-150. Springer, Heidelberg (1986)

11. Oort, F., Ueno, K.: Principally polarized abelian varieties of dimension two or three are Jacobian varieties. J. Fac. Sci. Univ. Tokyo Sect. IA Math. 20, 377-381 (1973)

12. Recillas, S.: Jacobians of curves with $\mathrm{g}^{1}{ }_{4}$ 's are the Prym's of trigonal curves. Bol. Soc. Mat. Mexicana (2) 19(1), 9-13 (1974)

13. Serre, J.-P.: Galois Cohomology. Springer Monographs in Mathematics. Springer, Heidelberg (2002)

14. Serre, J.-P.: Algebraic Curves and Class Fields. In: GTM, vol. 117, Springer, Heidelberg (1988)

15. Vakil, R.: Twelve points on the projective line, branched covers, and rational elliptic fibrations. Math. Ann. 320(1), 33-54 (2001)

16. Vélu, J.: Isogénies entre courbes elliptiques. C. R. Acad. Sci. Paris, Séries A 273, 305-347 (1971) 